\title{
Inappropriate use of naloxone in a hospital setting compromising patient safety: a quality improvement project
}

\author{
Authors: Jennifer Palfrey, ${ }^{A}$ Maria Mandel ${ }^{B}$ and Ruth Caulkin ${ }^{C}$
}

\section{Introduction}

Life threatening opioid toxicity is a rare but recognised complication of opioid use. In 2014, NHS England released a patient safety alert on inappropriate prescribing of naloxone in patients with chronic opioid use, citing two fatal cases. ${ }^{1}$

A retrospective audit (2017) of naloxone use in an acute hospital demonstrated that $90 \%(18 / 20)$ of administrations did not meet recommended standards. This prompted a quality improvement project to improve practice.

\section{Methods}

A trust guideline was developed using the Palliative care formulary and UK Medicines Information's naloxone evidence review. ${ }^{2,3}$ This was disseminated through education sessions and email updates. An alert was created on the trust electronic prescribing system which was visible to both prescribers and those administering the medication. This detailed the clinical parameters recommended before naloxone is to be given.

Following this, repeat data were collected retrospectively over 3 months for all adult hospital inpatients (excluding intensive treatment unit and emergency department) administered naloxone. Electronic clinical notes were reviewed for the reason for naloxone use, respiratory rate, oxygen saturation, level of consciousness and dose given.

\section{Results}

Repeat audit (2019) demonstrated three administrations of naloxone, two of which did not meet the guideline standard; an $89 \%$ reduction in inappropriate administrations of naloxone compared to baseline. The dose which was appropriately administered was also of the recommended dose.

\section{Discussion}

The clinical guideline introduced a 'traffic light system', grading severity of opioid toxicity based on clinical parameters. A closedloop flowchart was created, which provides clear descriptions of how to manage a patient on chronic opioids with suspected opioid toxicity. In addition, there are sources of help identified and

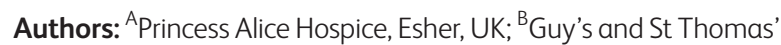
Hospital, London, UK; ${ }^{C}$ Chelsea and Westminster Hospital, London, UK recommendations for follow-up and continuous monitoring once the acute toxicity has been addressed.

A comprehensive education programme has enabled the learning from the audit and guideline to be disseminated to all grades of clinical staff through presentations at trust grand round, departmental education meetings, clinical governance meetings and junior doctor teaching sessions.

Ongoing efforts focus on continued dissemination of the guideline, particularly with rotation of clinical staff. Teaching on opioid toxicity and use of naloxone is now part of the annual teaching programme to trust internal medicine training and foundation year doctors.

The trust is due to undergo modernisation of its IT systems, including its electronic prescribing system. It is important that the prescribing alert for naloxone is maintained on future prescribing systems.

\section{Conclusion}

There has been a marked decrease in inappropriate administrations of naloxone in patients with chronic opioid use since the interventions. However, continued work is needed as a reduced conscious level often triggered naloxone use despite absence of respiratory depression or hypoxia.

Education sessions and update emails were helpful to highlight the concerns raised in the initial audit and to familiarise staff with the new guideline. These will remain important due to the rotation of staff however the prescribing alert was more effective in establishing sustained change in practice as it is visible and current at the time of prescribing and administration of naloxone.

\section{Conflicts of interest}

None declared.

\section{References}

1 NHS England. Patient safety alert: Risk of distress and death from inappropriate doses of naloxone in patients on long-term opioid/ opiate treatment. NHS, 2014.

2 Twycross R, Wilcock A, Howard P et al (eds). Palliative care formulary. Nottingham: Palliativedrugs.com, 2014.

3 UK Medicines Information. Medicines Q\&As: What naloxone doses should be used in adults to reverse urgently the effects opioids or opiates? UKMi, 2017. www.sps.nhs.uk/wp-content/ uploads/2015/11/UKMi_QA-_Naloxone-dosing_Aug-17_FINAL.pdf 